\title{
Fusion of QuickBird Multi-sensor Images using Different Techniques
}

\author{
ElSherif A. M. ${ }^{1}$, Afifi H. A. ${ }^{2}$
}

1. Civil Engineering, Public Works Department, Faculty of Engineering, Tanta University
,Egyt

2. Civil Engineering, Public Works Department, Faculty of Engineering, Tanta University

,Egypt

\begin{abstract}
Earth observation satellites such as QuickBird provide panchromatic (PAN) images at high spatial resolution and multispectral (MS) images at high spectral resolution but low spatial resolution. Actually, due to sensor limitations and technical restrictions the instruments are not capable of directly providing an image with high spatial and spectral resolutions. The most efficient and economic way to produce high spatial resolution MS images is by applying image fusion techniques to merge the high spatial information of the PAN image with the high spectral information of the MS image. This paper describes and examines six of the most widely used fusion techniques to merge two sets of QuickBird PAN and MS images covering agricultural and urban areas in Tanta and Alexandria cities, Egypt. The applied fusion techniques utilize different spectral and spatial transformations, they are (1) Intensity-Hue-saturation (IHS), (2) Principle Component Analysis (PCA), (3) High Pass Filter (HPF), (4) Mallat Wavelet Addition (MWA), (5) IHS with Area Model (IHS+Area), and (6) University of New Brunswick fusion model (UNB). The purpose of this study is to evaluate and compare the spectral and spatial qualities of the fused images due to applying different fusion techniques. The fused images are compared visually and statistically to the original PAN and MS images. The results revealed that the (IHS+Area) method has considerably improved the spectral quality while preserving the spatial information of the original PAN image. However, the improvement of the spectral quality due to applying a certain technique means the deterioration of its spatial quality. Thus, the selection of an appropriate fusion technique depends mainly on the application requirements
\end{abstract}

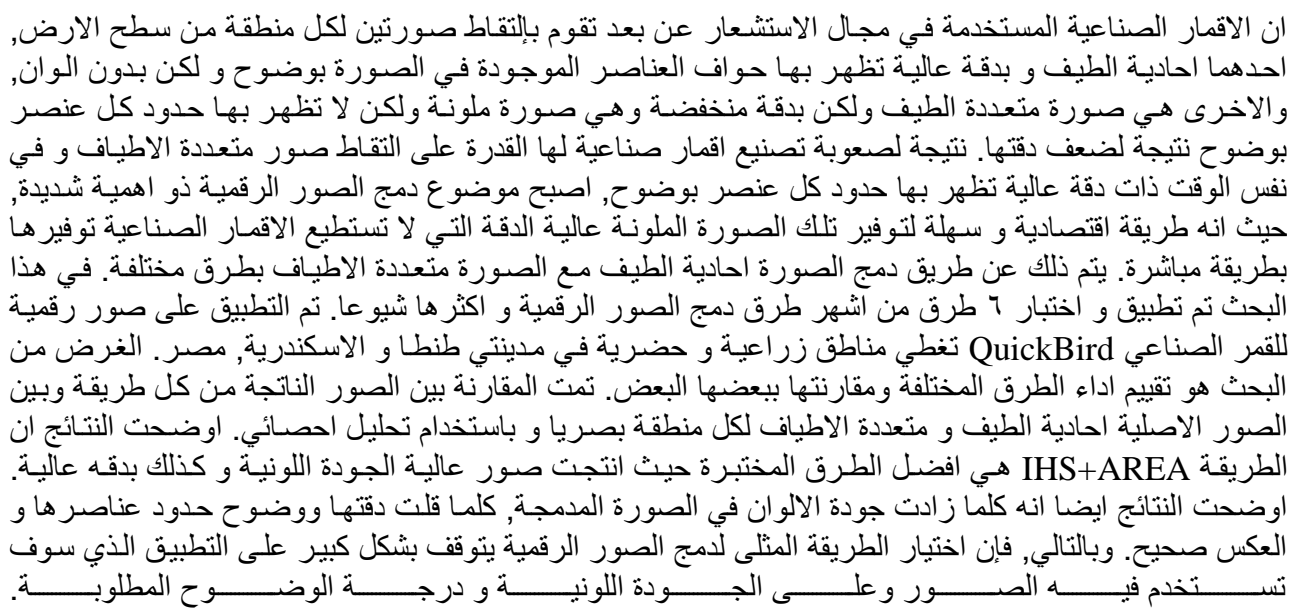

\section{INTRODUCTION}

Earth observation satellites provide an increasing amount of images at different spatial, temporal, radiometric, and spectral resolutions. QuickBird satellite provides PAN images at $0.6 \mathrm{~m}$ ground resolution and MS images in four bands (blue, green, red, and near infrared) at $2.4 \mathrm{~m}$ ground resolution.
Due to sensor limitations and technical restrictions, QuickBird system is not capable of directly providing an image with high spatial and spectral resolutions. Moreover, many remote sensing applications such as classification, change detection, feature recognition and mapping of urban areas require images that simultaneously have high spatial and high spectral resolutions. Therefore, the most efficient and 
economic way to produce high spatial resolution MS images is by applying image fusion techniques to merge the spatial details of PAN images with the spectral information of MS images.

During the last two decades, several fusion techniques have been developed and reported in scientific papers [Wald et al., 1997; Pohl and Van Genderen , 1998; Ranchin and Wald, 2000; Zhang, 2002, 2004; Tu et al., 2002, 2004; Svab and Ostir, 2006; Ehlers et al., 2010; Firouz et al., 2011; Afify, 2012]. However, of the most widely used techniques to perform the image fusion of satellite imagery are (1) Intensity-Hue-saturation (IHS), (2) Principle Component Analysis (PCA), (3) High Pass Filter (HPF), (4) Mallat Wavelet Addition (MWA), (5) IHS with Area Model (IHS+Area), in addition to (6) University of New Brunswick fusion model (UNB). The IHS and PCA fusion techniques are based on the spectral transformations of RGB color space to IHS and PCA color spaces respectively. The HPF and MWA fusion techniques utilize spatial transformations to separate the high frequency information (spatial details) of the PAN image and then inject it to the low frequency information (spectral information) of the MS image. The IHS+Area fusion technique is a modified IHS with different weighting parameters for each band of the MS image. The UNB fusion model uses the least square algorithm to generate a synthesized image from the MS bands that is used to generate the fused MS image. Regarding the different theoretical principle for each of these six image fusion techniques it is worth to study the effect of each on the spatial and spectral qualities of the resulted fused images.

In this paper, these six image fusion techniques are presented and applied to merge two sets of QuickBird PAN and MS images covering different features of Tanta and Alexandria cities in Egypt. The aim is to compare their performance and to assess the effect of each fusion technique on the spatial and spectral properties of the fused images. The resulted fused images are inspected visually and evaluated statistically to assess their spectral and spatial qualities. The processing steps of this study were performed by the aid of PCI, ENVI and ERDAS digital image processing software packages.

\section{Study Site and Data Sets}

Two data sets of QuickBird images were used. Each data set comprises a PAN and a MS subscenes. The first set was acquired on September 3, 2010 covering an agricultural area of Tanta city, ElGharbiya, Egypt. The second set was acquired on May 6, 2007 covering a city area with different urban features of Alexandria, Egypt. For each set, the MS image was registered to its corresponding PAN image. The ground control points (GCPs) were automatically collected between both PAN and MS images using AUTOGCP module in PCI that is based on the normalized cross-correlation approach. Then, the second order polynomial and the cubic convolution resampling technique were applied to determine the gray value of each pixel in the registered MS image. The accuracy of the registration process is less than quarter a pixel for both data sets. After the geometric registration, the PAN subscene was cut out from the PAN image with a size of 1024 pixels by 1024 pixels, $0.6 \mathrm{~m}$ each, and the MS subscene was cut out from the MS image with a size of 256 pixels by 256 pixels, $2.4 \mathrm{~m}$ each as shown in figures (1 and 2).

\section{Image Fusion Techniques}

\subsection{IHS fusion method}

The IHS fusion method uses three low resolution MS bands and transforms them from RGB color space to IHS color space which offers the advantage that the separate components outline certain color properties as follows, [Firouz et al., 2011].

$\left[\begin{array}{l}\mathrm{I} \\ \mathrm{v}_{1} \\ \mathrm{v}_{2}\end{array}\right]=\left[\begin{array}{ccc}1 / 3 & 1 / 3 & 1 / 3 \\ -\sqrt{2} / 6 & -\sqrt{2} / 6 & 2 \sqrt{2} / 6 \\ 1 / \sqrt{2} & -1 / \sqrt{2} & 0\end{array}\right]\left[\begin{array}{l}\mathrm{R} \\ \mathrm{G} \\ \mathrm{B}\end{array}\right]$

Where, $\mathrm{H}=\tan ^{-1}\left(\mathrm{v}_{2} / \mathrm{v}_{1}\right)$, and $\mathrm{S}=\sqrt{\mathrm{v}_{1}{ }^{2}+\mathrm{v}_{2}{ }^{2}}$

The intensity component $\mathrm{I}$ is replaced by the PAN image. Then the composition (Pan, $\mathrm{H}$, and $\mathrm{S}$ ) is transformed back into original RGB color space. To reduce the multiplication and addition operations, a fast IHS (FIHS) fusion can be implemented according to equation (2)

$$
\begin{aligned}
{\left[\begin{array}{l}
\mathrm{R}^{\prime} \\
\mathrm{G}^{\prime} \\
\mathrm{B}^{\prime}
\end{array}\right]=} & {\left[\begin{array}{ccc}
1 & -1 / \sqrt{2} & 1 / \sqrt{2} \\
1 & -1 / \sqrt{2} & -1 / \sqrt{2} \\
1 & \sqrt{2} & 0
\end{array}\right]\left[\begin{array}{l}
\mathrm{PAN} \\
\mathrm{V}_{1} \\
\mathrm{~V}_{2}
\end{array}\right] } \\
= & {\left[\begin{array}{ccc}
1 & -1 / \sqrt{2} & 1 / \sqrt{2} \\
1 & -1 / \sqrt{2} & -1 / \sqrt{2} \\
1 & \sqrt{2} & 0
\end{array}\right]\left[\begin{array}{l}
\mathrm{I}+(\mathrm{PAN}-\mathrm{I}) \\
\mathrm{V}_{1} \\
\mathrm{~V}_{2}
\end{array}\right] } \\
= & {\left[\begin{array}{l}
\mathrm{R}+(\mathrm{PAN}-\mathrm{I}) \\
\mathrm{G}+(\mathrm{PAN}-\mathrm{I}) \\
\mathrm{B}+(\mathrm{PAN}-\mathrm{I})
\end{array}\right]=\left[\begin{array}{l}
\mathrm{R}+\delta \\
\mathrm{G}+\delta \\
\mathrm{B}+\delta
\end{array}\right] }
\end{aligned}
$$

Where, R', G', B' are the fused images, and $\delta=($ PAN - I)

Equation (2) states that the fused images R', G', and $\mathrm{B}^{\prime}$ can be easily obtained by adding the difference image between Pan and I to the original MS images. Generally, as the difference $(\delta)$ increases, more color distortion is expected to appear in the fused image as a result of mismatches, that is 
the Pan and I images are spectrally dissimilar [Zhang, 2004].

\subsection{PCA fusion method}

The PCA fusion method is based on the spectral transformation from RGB color space to PCA color space to transform the original correlated MS bands into a set of uncorrelated principal components (PCs). The PCs will be ranked in terms of the variance they explain from the original bands [Ricotta 1999; Eastman 2006]. So, the first two or three PCs usually contain most of the information (over than 95\%) involved in the original MS bands.

The PCA fusion technique assumes that the first component PC1 contains the overall scene luminance [Pohl et al., 1998] and is similar to the data presented in the PAN image [Chavez et al., 1991]. Based on the previous assumption, the PAN image substitutes the PC1 after being scaled to match the histogram of the PC1 component. Finally, an inverse PCA transform is applied to the matched PAN image and the rest of PC components to obtain the fused images.

\subsection{HPF fusion method}

[Chavez et al, 1991] introduced the High Pass Filtering method for panchromatic and multispectral image fusion. In the high pass filtering fusion technique, the high frequencies in the panchromatic image are extracted using a high pass filter, and then are introduced into the original MS bands by simple addition. Although the multispectral bands aren't equally correlated to the panchromatic image, the high frequencies are introduced equally to them. The high pass filter can be expressed as the following:

$$
\left[\begin{array}{lll}
-1 & -1 & -1 \\
-1 & M & -1 \\
-1 & -1 & -1
\end{array}\right]
$$

As $M$ resembles the mid value in the filter and varies according to the purpose of fusion and the amount of high frequencies to be extracted from the PAN image. The higher $M$ value is, the higher the spatial information extracted from the PAN image and presented in the fused image. In this study a value of $M=24$ is considered as a well-suited mid value in the filter to merge Quickbird images.

\subsection{MWA fusion method}

Multiresolution wavelet decomposition provides a powerful tool to separate the spectral content of an image from the spatial content. Mallat wavelet [Gonzalez et al., 2004; Amolins et al., 2007] is one of the most common wavelet models used in image fusion. It contains a bank of high and low pass filters which decomposes the input image into four images with less resolution; one approximation image and three (horizontal, vertical, and diagonal) detail images.
The Mallat wavelet addition fusion technique was performed according to the scheme shown in figure (3). After the geometric registration, each MS band was resampled so that it has the same pixel size as the PAN image. The PAN image was then histogram matched to the MS band and the Mallat wavelet decomposition was applied twice times to each of the matched PAN and the resampled MS band. Then the detail images of the PAN image are added to the detail images of the MS band. Finally, Mallat's inverse wavelet transform was applied twice times using the approximation image of the MS band and the added detail images of the PAN image and MS band resulting in a fused band.

\section{5.(IHS+Area) fusion method}

The IHS+Area fusion technique is based on the fast IHS (FIHS) fusion technique previously described in equation (2). The aim is to derive a new modified intensity image $I$ that minimizes the radiance difference $(\delta)$ between Pan and I images. The achievement of the IHS fusion technique using equation (2) allows the extension of traditional threeorder transformation to an arbitrary order. This means that the NIR band can be included in the definition of I component [Tu et al., 2004] and the (FIHS) fusion can be represented as follows:

$$
\left[\begin{array}{c}
\mathrm{R}^{\prime} \\
\mathrm{G}^{\prime} \\
\mathrm{B}^{\prime} \\
\mathrm{NIR}
\end{array}\right]=\left[\begin{array}{c}
\mathrm{R}+\delta_{1} \\
\mathrm{G}+\delta_{1} \\
\mathrm{~B}+\delta_{1} \\
\mathrm{NIR}+\delta_{1}
\end{array}\right]
$$

Where, $\mathrm{I}_{1}=(\mathrm{R}+\mathrm{G}+\mathrm{B}+\mathrm{NIR}) / 4$

$\delta_{1}=\left(\operatorname{Pan}-\mathrm{I}_{1}\right)$

Equation (4) stated that in FIHS all the MS bands are equally considered to derive the new intensity component. However, taking into consideration that the measured energy in an individual channel is sum (integral) of incoming radiation and relative spectral sensitivity, it is theoretically possible to obtain the values in the PAN band with the summation of respective spectral bands. Unfortunately the spectral response curve of the PAN band does not completely cover the MS bands.

Afify, (2012) suggested using different weighting parameters determined according to the intersection area between the spectral response curve of each MS band and that of the PAN band to assign the contribution of each MS band in the derived I component. The weighting parameter for a certain MS band was assigned as the ratio between the intersection area of that band to the sum of intersection areas of all MS bands. A new intensity component can be determined after introducing the calculated weighting parameters for all the bands of QuickBird as follows:

$\mathrm{I}_{\text {new }}=0.111(\mathrm{~B})+0.264(\mathrm{G})+0.237(\mathrm{R})+0.388(\mathrm{NIR})$ 
In addition an appropriate tradeoff parameter in the interval $[0,1]$ was then used to improve the spectral characteristics of the fused images. Hence, the IHS+Area fusion technique can be expressed as follows [Afify, 2012]:

$$
\left[\begin{array}{c}
R^{\prime} \\
G^{\prime} \\
B^{\prime} \\
N I R^{\prime}
\end{array}\right]=\left[\begin{array}{c}
R+t \cdot\left(P A N-I_{\text {new }}\right) \\
G+t \cdot\left(P A N-I_{\text {new }}\right) \\
B+t \cdot\left(P A N-I_{\text {new }}\right) \\
N I R+t \cdot\left(P A N-I_{\text {new }}\right)
\end{array}\right]
$$

In this study the appropriate tradeoff parameter $(\mathrm{t})$ is taken equal to 0.7 .

\subsection{UNB fusion method}

The UNB fusion algorithm was developed by Zhang, (2002). It was reported by the author that the new fusion approach is capable of successfully preserve both the spectral and spatial characteristics in the fusion process and produce a high-resolution multispectral image with very low color distortion, especially for the multispectral bands that provide good frequency (wavelength) overlap with the corresponding panchromatic image. Although the fusion algorithm and detailed procedure is kept locked and unavailable by the developer, the algorithm was available as pan-sharpening module in the PCI digital image processing software from Geomatica, Ottawa, Canada.

\section{Experiements and Results}

After registering the MS image of each set to its corresponding PAN image, the MS images were upsampled using cubic convolution so that the pixel size of MS bands equals that of the PAN image (0.6 $\mathrm{m})$. Then the fusion techniques were applied to merge the two data sets of QuickBird images. Figures (1 and 2) show the fused images for the two data sets. To statistically evaluate the spectral quality of the fused images, they were first degraded to their original spatial resolution $(2.4 \mathrm{~m})$ using cubic convolution resampling, and then compared to the original MS bands by computing the following quantitative parameters:

1-The correlation coefficients (CCs) between the fused bands and the original MS bands where,

Where

$$
C C(A / B)=\frac{\sum_{i=1}^{n}\left(A_{i}-\bar{A}\right)\left(B_{i}-\bar{B}\right)}{\sqrt{\sum_{i=1}^{n}\left(A_{i}-\bar{A}\right) \sum_{i=1}^{n}\left(B_{i}-\bar{B}\right)}}
$$

$$
\begin{aligned}
& \mathrm{A}_{\mathrm{i}} \text { and } \mathrm{B}_{\mathrm{i}} \quad \begin{array}{l}
=\text { the pixel values of the original } \\
\text { and fused images. }
\end{array} \\
& \overline{\mathrm{A}} \text { and } \overline{\mathrm{B}} \quad \begin{array}{l}
=\text { the mean values of the original } \\
\text { and fused images. }
\end{array}
\end{aligned}
$$

\section{2-ERGAS (Erreur Relative Globale} Adimensionnelle de Synthèse) is a simplified quantity that summarizes the errors in all the bands. The lower the ERGAS value, the better the spectral quality of the fused images. The ERGAS index for the fusion is expressed as follows:

$$
\mathrm{ERGAS}=100 \frac{\mathrm{h}}{1} \sqrt{\frac{1}{\mathrm{~N}} \sum_{\mathrm{k}=1}^{\mathrm{N}} \frac{\operatorname{RMSE}^{2}\left(\mathrm{~A}_{\mathrm{k}}\right)}{\overline{\mathrm{A}}_{\mathrm{k}}{ }^{2}}}
$$

Where,

$\mathrm{h}=$ the resolution of the high spatial resolution image.

$1=$ the resolution of the low spatial resolution image. $\mathrm{N}=$ number of bands.

$\overline{\mathrm{A}}_{\mathrm{k}}=$ the mean values of the original band $\mathrm{k}$.

RMSE $(\mathrm{A})=$ the root mean square error that can be computed as follows:

$\operatorname{RMSE}(\mathrm{A})=\sqrt{\frac{\sum_{\mathrm{i}=1}^{\mathrm{n}}\left(\mathrm{A}_{\mathrm{i}}-\mathrm{B}_{\mathrm{i}}\right)^{2}}{\mathrm{n}}}$

$A_{i}$ and $B_{i}=$ the pixel values of the original and fused images.

$\mathrm{n}=$ number of pixels.

Tables (1 and 2) show the correlation coefficients and the ERGAS index values for the two data sets

To evaluate the spatial quality of the fused images, the Pan and fused images were filtered using the high pass Laplacian filter then the correlation coefficients between the filtered Pan and the filtered fused images were computed [Zhou et al., 1998]. Then the average of the correlation coefficients is calculated and provided in tables (1 and 2) to determine the overall spatial quality of the fused image. The high correlation coefficients indicate that most of the spatial information of the PAN image was injected into the MS image during the fusion process. Tables (1 and 2) show the correlation coefficients between the filtered PAN and the filtered fused images by different methods for the two data sets.

\section{Analysis of Results}

From Tables (1 and 2), it can be noted that Mallat wavelet addition (MWA) has provided the highest spectral quality followed by the IHS+Area fusion technique. Both techniques provided average spectral correlation coefficients (CCs) ranging from 95\% to $98.5 \%$ for agricultural and urban areas of the two data sets. Spectrally, they are followed by the UNB fusion technique with (CCs) of $89 \%$. HPF and PCA fusion followed the UNB fusion technique and finally with the least spectral quality especially in the blue band of Tanta agricultural area (data set 1) is the traditional IHS fusion technique. 
The reason of this significant color distortions produced by IHS fusion technique is the differences in the spectral response curves between the PAN band and each of the MS bands. For QuickBird sensors, the spectral response curve of the PAN band poorly covers that of the blue band and also with extremely low sensitivity. Another reason is that, in IHS fusion, the NIR band is not considered in the definition of the I image that introduces high color distortion especially in the vegetation areas. Visually the color distortion is clear and the green cover trends to the blue color as shown in figure (1-c).

The spectral quality obtained using the PCA and HPF is higher than that obtained using the traditional IHS method but still not satisfactory. These improvements can be referred to the consideration of the NIR band in the spectral transformation from RGB color space to PCA color space and also to the injection of high frequency details of the PAN image to the original MS bands without altering their original grey values in HPF fusion technique. Visually, the colors of different features are closer to the original MS bands, but still a significant color distortion in the green parts is presented in the PCA fused image, while the HPF and UNB fused images contain the correct color combinations for each feature but in a paler color tone than the original MS image. It is also worth to be noted that the fusion quality in the agricultural data set is significantly lower than that of the urban data set.

The MWA and IHS+Area produced fused images almost spectrally undistorted in the two data sets. There is no significant spectral difference between the fused images of the two models visually. However, the statistical parameters indicated that the MWA fused images are of higher spectral quality than the IHS+Area fused images. The MWA fusion model delivered this superb fusion performance due to its procedure in which the detail images of the MS bands components are added to and not replaced by those of the Pan image. Thus, all the detail information of the MS bands is preserved; For the IHS+Area, the reasons of the high spectral quality produced can be referred to the introduction of the NIR band into the derived intensity layer and the usage of different weighting parameters according to the overlap area between the spectral response curve of each MS band and the PAN image. Another reason is the usage of a tradeoff parameter that controls spatial/spectral information introduced into the fused bands.

The computed values of ERGAS index for different fusion techniques provided parallel results to that obtained using the CCs. Among the tested fusion techniques, the MWA fusion is spectrally the superior fusion technique since it provided the least ERGAS values for both data sets (1.9100 for agricultural area and 2.2327 for urban area).
All the applied fusion techniques have introduced spatial details but the degree of sharpness varies in the fused images. The edges and the field's boundaries in Tanta agricultural area and the small objects like cars in Alexandria urban area are clearly visible in the fused images. Among all the methods, the PCA method has produced the highest spatial quality followed by the HPF fusion. The UNB and IHS+Area models provided fused images with almost the same spatial quality slightly below that of the HPF fusion technique. They are followed by the IHS fusion technique that produced lower spatial quality especially in the vegetation areas. MWA provided the least spatial quality with significant deterioration below the IHS fusion even though it produced the highest spectral quality.

The visual inspection goes parallel to the statistical evaluation and shows that the PCA and HPF fusion models produced high spatial quality in the two data sets. However, the statistical analysis indicated that the UNB fused images are smoother than the PCA and HPF fused images, but the visual inspection indicates otherwise and shows that the UNB fused images are as sharp as the HPF, PCA, and the original PAN images

Generally, the obtained spatial quality due to applying a certain fusion method is always higher in Alexandria site (urban area) than in Tanta site (agricultural area). This can be attributed to the nature of the spatial details of different land cover classes in the area under consideration. However the results obtained due to applying the IHS+Area and UNB fusion techniques are considerably stable regarding the scene nature and land cover classes.

\section{Conclusions}

This study showed the capability of different fusion techniques to successfully produce high spatial resolution multispectral images with various degrees of spatial and spectral qualities. The traditional IHS technique has provided fused images with significant color distortions. The MWA fusion model superbly preserves the spectral quality of the fused images in different data sets and land covers. However, that superb spectral quality is in expense of the spatial quality.

Using the PCA, HPF, and UNB techniques improved the spectral quality better than that produced by IHS fusion but still less than that obtained using IHS+Area or MWA fusion techniques. These improvements in the spectral quality are referred to the consideration of the near infrared band in fusion process.

Regarding its fast and simple computing capability, the IHS+Area is capable of preserving high spectral and spatial quality whatever the type of land cover is. It is the only fusion technique among the tested techniques that provided fused images 
having correlation coefficients with the originals higher than $95 \%$ both spatially and spectrally. Therefore, the IHS+Area technique is chosen as the best fusion model among the tested fusion techniques.

This study demonstrated that the improvement of the spectral quality due to applying a certain technique means the deterioration of the spatial quality and vise versa. However, the selection of an appropriate fusion technique depends mainly on the application requirements that might be an enhanced naturalcolored image for better visualization, a sharper and greater detail in color image for more accurate mapping or a classification oriented fused image.

\section{References}

Amolins, K., Zhang, Y., Dare, P., (2007). Waveletbased image fusion techniques - an introduction, review and comparison, ISPRS Journal of Photogrammetry and Remote Sensing, 62(4), 249263.

Afify, H. A., (2012). A New Intensity-HueSaturation Based Method for Fusing High Resolution Satellite Images, International Journal of Geoinformatics, IJG, Vol. 8, No. 4, December 2012, pp 1-14

Chavez, P.S., Stuart, J., Sides, C., Anderson, J.A., (1991). Comparison of three different methods to merge multiresolution and multispectral data: Landsat TM \& SPOT Panchromatic. Photogrammetric Engineering and Remote Sensing, 57, 295-303.

Eastman, J. R. (2006). Idrisi Andes. Guide to GIS and image processing. Clarks Laboratories.

Ehlers, M., Klonus, S., Johan, P., strand, A., and Rosso, P., (2010). Multi-sensor image fusion for Pan sharpening in remote sensing". International Journal of Image and Data Fusion, 1(1), 25-45.

Firouz, A. W., Kalyankar, N. V., Al-Zuky, A. A., (2011). The IHS transformations based image fusion, Journal of Global Research in Computer Science (JGRCS), 2(5) May 2011, Available Online at www.jgrcs.info 70 .

González Audícana, M., Saleta, J.L., García Catalán, R., and García, R., (2004). Fusion of multispectral and panchromatic images using improved IHS and PCA mergers based on wavelet decomposition, IEEE Transactions on Geoscience and Remote Sensing, 42(6), 1291-1299.

Pohl, C. and Van Genderen, J. L., (1998). Multisensor image fusion in remote sensing: concepts, methods and applications (Review Article), International Journal Of Remote Sensing, 19(5), 823854.

Ranchin, T., and Wald, L., (2000). Fusion of high spatial and spectral resolution images: The ARSIS concept and its implementation, Photogrammetric Engineering \& Remote Sensing, 66(1), 49-61.

Ricotta C, Avena G.C.F. Volpe (1999). The influence of principal component analysis on the spatial structure of multispectral dataset, International Journal for Remote Sensing Volume 20 No 17 page 3367-3376.

ŠVab A.and Oštir K., (2006). High-resolution image fusion: Methods to preserve spectral and spatial resolution, Photogrammetric Engineering \& Remote Sensing, 72(5), 565-572.

Tu, T.M., Su, S., Shyu, H., and Huang, P.S., (2002). A new look at IHS like image fusion methods, Information Fusion, 2(3), 177-186.

Tu, T.-M., Huang, P.S., Hung, C.-L., and Chang, C.-P., (2004). A fast intensity-hue-saturation fusion technique with spectral adjustment for IKONOS imagery, IEEE Geoscience and Remote Sensing Letters, 1(4), 309-312.

Wald, L., Ranchin, T., and Mangolini, M., (1997). Fusion of satellite images of different spatial resolutions: Assessing the quality of resulting images, Photogrammetric Engineering \& Remote Sensing, 63(6), 691-699.

Zhang Y., (2002). Problems in the fusion of commercial high-resolution satelitte images as well as Landsat 7 images and initial solutions, International Archives of Photogrammetry and Remote Sensing (IAPRS), Volume 34, Part 4

Zhang, Y., (2004). Understanding image fusion, Photorammetric Engineering \& Remote Sensing, 70(6), 657-661.

Zhou, J., Civco, D.L. and Silander, J.A., (1998). A wavelet transform method to merge Landsat TM and SPOT panchromatic data. International Journal of Remote Sensing, $\quad 19, \quad 743-757$. 
ElSherif A. M., Afifi H. A" Fusion of QuickBird Multi-sensor Images using Different .........'

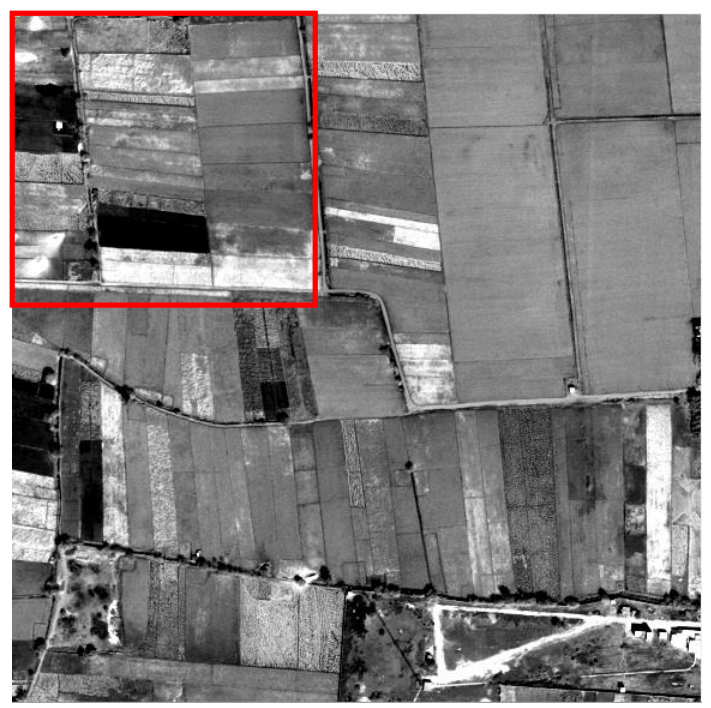

(a)

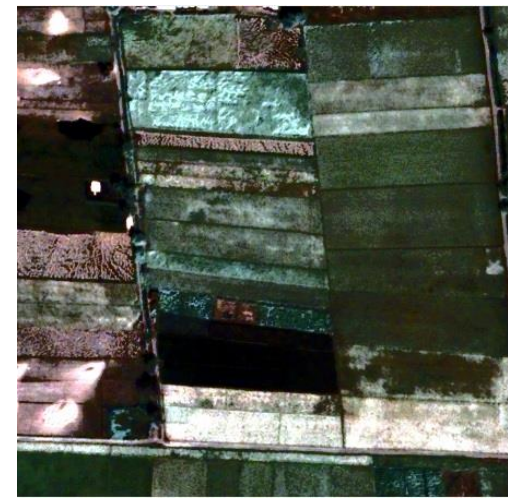

(c)

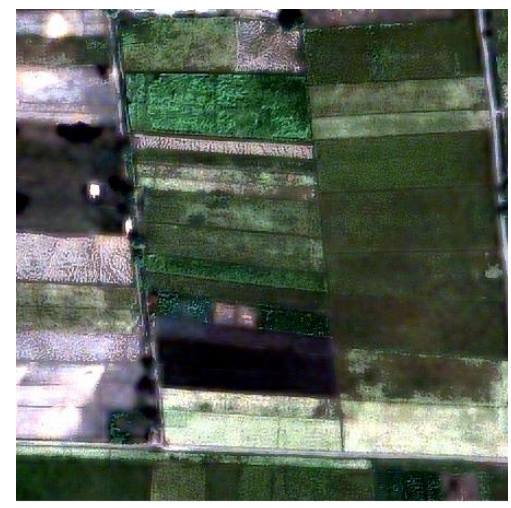

(f)

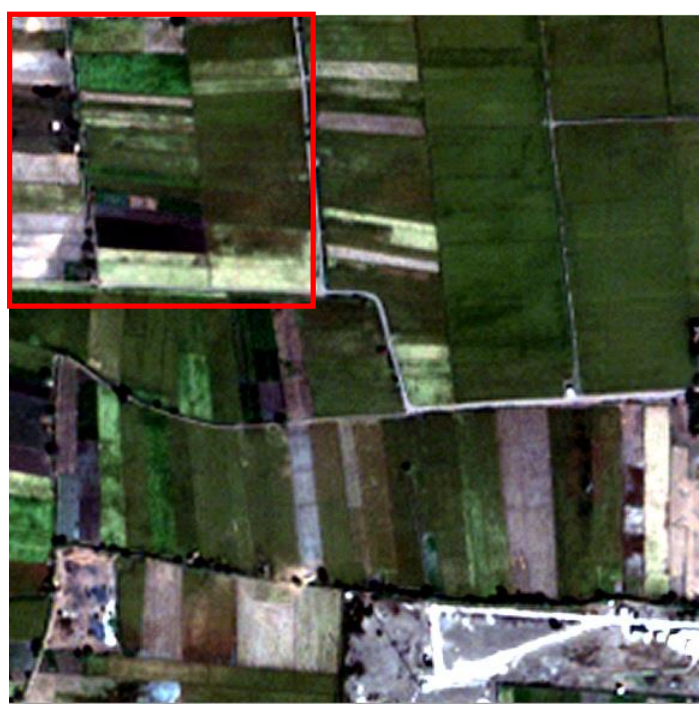

(b)

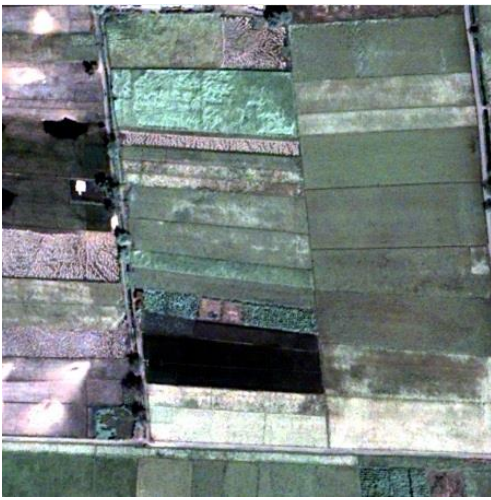

(d)

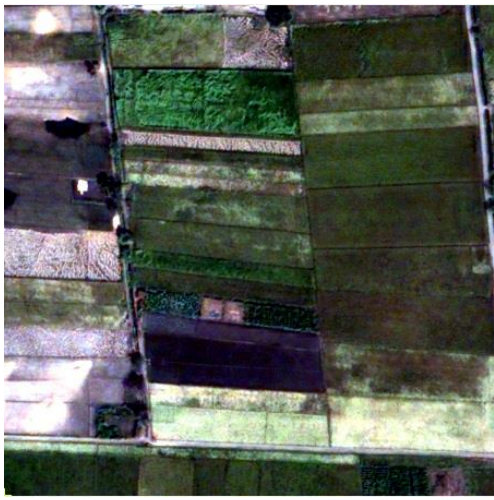

$(\mathrm{g})$

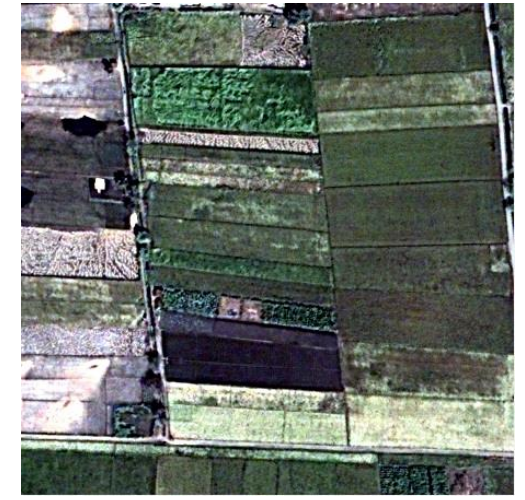

(e)

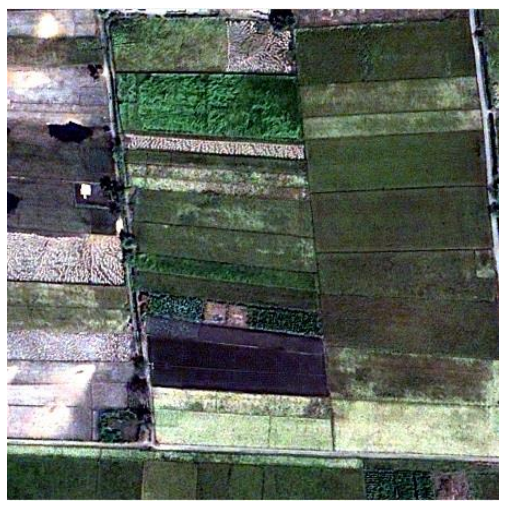

(h)

Figure 1: Original images and results of fusion methods for Tanta site,

(a) Original PAN, (b) Original MS, (c) IHS, (d) PCA, (e) HPF, (f) MWA, (g) IHS+Area, (h) UNB 


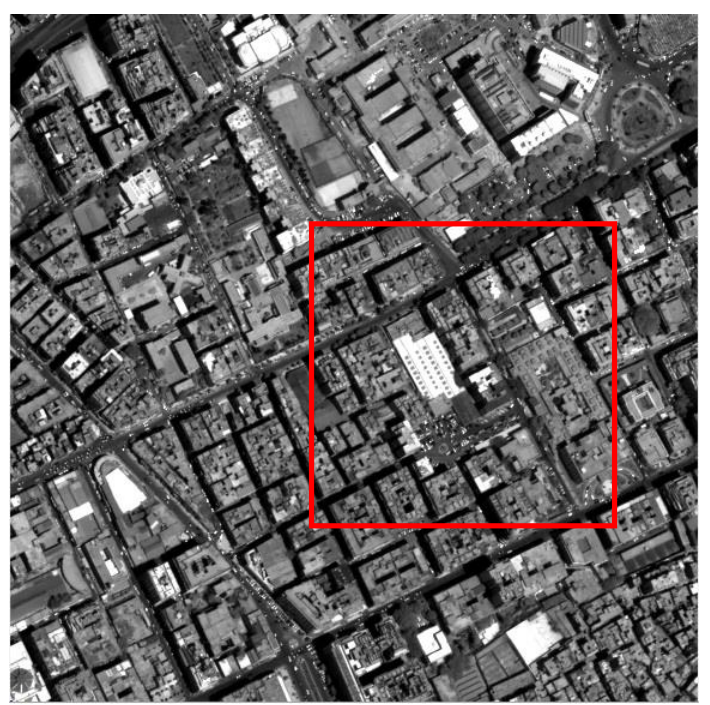

(a)

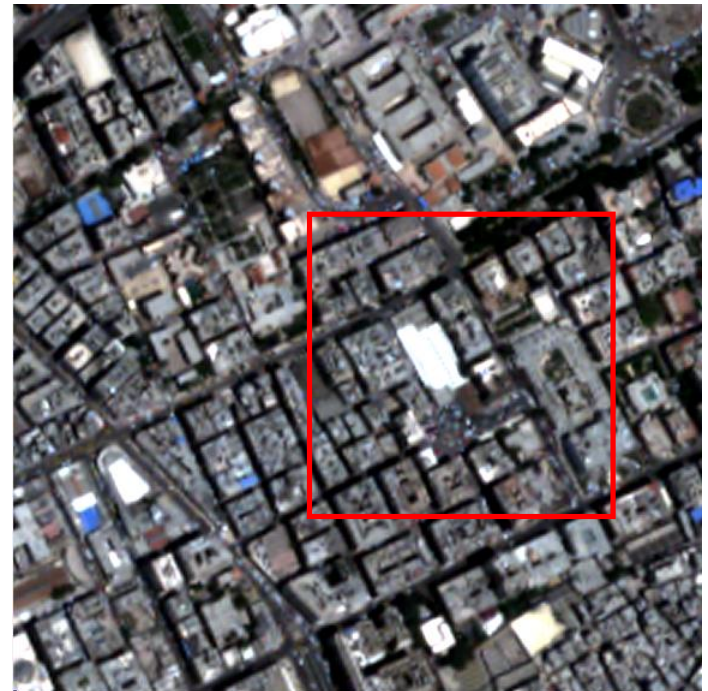

(b)

Figure 2: Original images and results of fusion methods for Alexandria site,

(a) Original PAN, (b) Original MS, (c) IHS, (d) PCA, (e) HPF, (f) MWA, (g) IHS+Area, (h) UNB

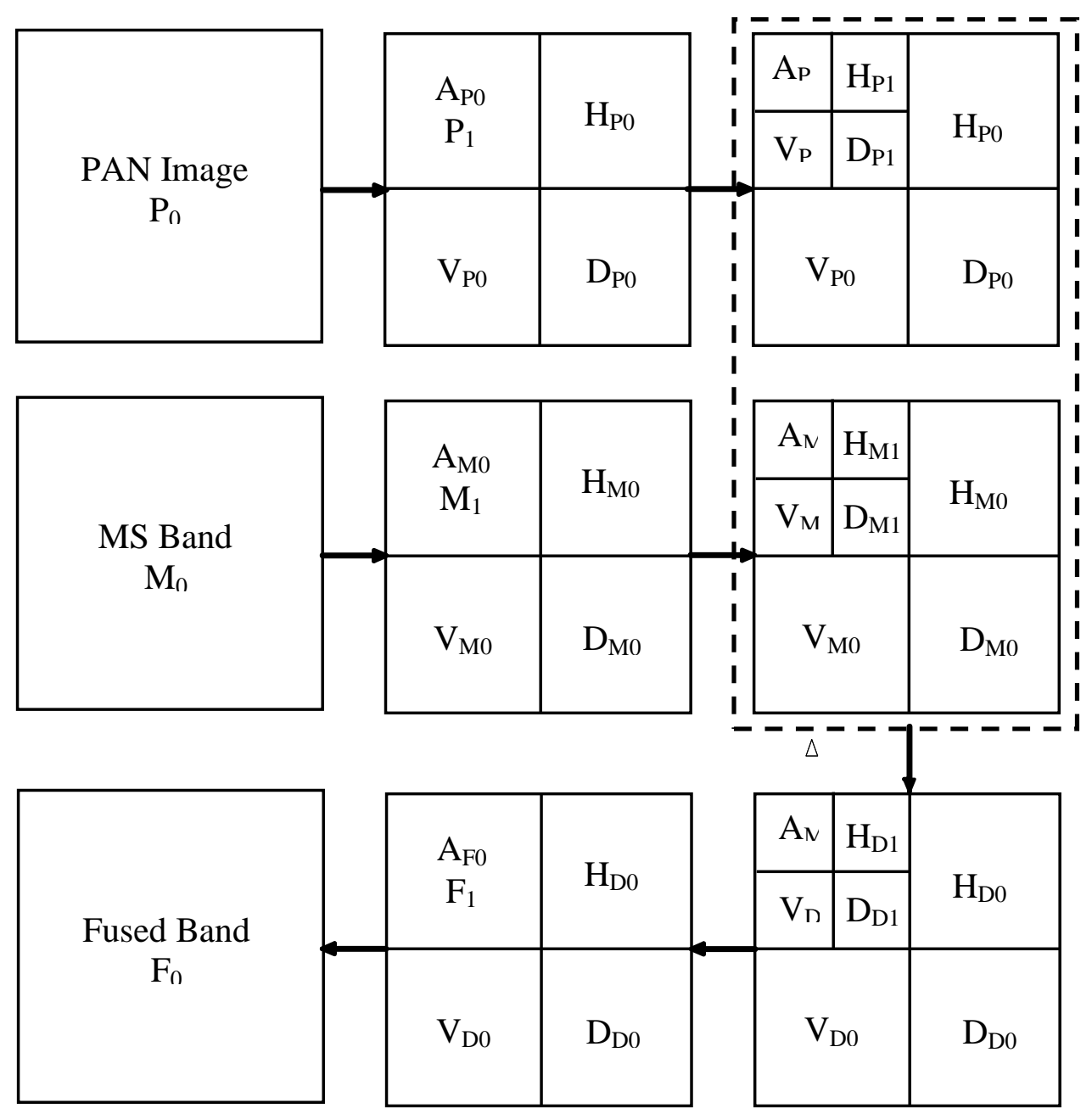

$\mathrm{H}_{\mathrm{D} 0}=\left(\mathrm{H}_{\mathrm{M} 0}+\mathrm{H}_{\mathrm{P} 0}\right), \mathrm{V}_{\mathrm{D} 0}=\left(\mathrm{V}_{\mathrm{M} 0}+\mathrm{V}_{\mathrm{P} 0}\right), \mathrm{D}_{\mathrm{D} 0}=\left(\mathrm{D}_{\mathrm{M} 0}+\mathrm{D}_{\mathrm{P} 0}\right), \mathrm{H}_{\mathrm{D} 1}=\left(\mathrm{H}_{\mathrm{M} 1}+\mathrm{H}_{\mathrm{P} 1}\right), \mathrm{V}_{\mathrm{D} 1}=\left(\mathrm{V}_{\mathrm{M} 1}+\mathrm{V}_{\mathrm{P} 1}\right), \mathrm{D}_{\mathrm{D} 1}=\left(\mathrm{D}_{\mathrm{M} 1}+\mathrm{D}_{\mathrm{P} 1}\right)$

Figure (3): The scheme of Mallat wavelet addition fusion technique 


\begin{tabular}{|l|c|c|c|c|c|c||}
\hline Criterion & IHS & PCA & HPF & MWA & IHS +Area & UNB \\
\hline CC Red & 0.7145 & 0.7789 & 0.8793 & 0.9778 & 0.9801 & 0.9153 \\
\hline CC Green & 0.6303 & 0.7378 & 0.8541 & 0.9684 & 0.9645 & 0.8906 \\
\hline CC Blue & 0.4935 & 0.6943 & 0.8434 & 0.9634 & 0.9593 & 0.8882 \\
\hline \hline CC (Ave) & 0.6128 & 0.7370 & 0.8589 & 0.9699 & 0.9680 & 0.8980 \\
\hline ERGAS & 8.0818 & 6.1193 & 4.2887 & 1.9100 & 1.9545 & 3.6026 \\
\hline \hline CC (HPF) & 0.8444 & 0.9827 & 0.9819 & 0.7926 & 0.9633 & 0.9667 \\
\hline
\end{tabular}

Table 1: Evaluation criteria (the correlation coefficients and ERGAS) for Tanta data set 1 (Agricultural area)

\begin{tabular}{|l|c|c|c|c|c|c||}
\hline \hline Criterion & IHS & PCA & HPF & MWA & IHS +Area & UNB \\
\hline CC Red & 0.9143 & 0.9195 & 0.9284 & 0.9854 & 0.9651 & 0.8851 \\
\hline CC Green & 0.9205 & 0.9237 & 0.9319 & 0.9864 & 0.9679 & 0.8896 \\
\hline CC Blue & 0.8227 & 0.9248 & 0.9289 & 0.9792 & 0.9261 & 0.8875 \\
\hline \hline CC (Ave) & 0.8858 & 0.9227 & 0.9297 & 0.9837 & 0.9530 & 0.8874 \\
\hline ERGAS & 6.0693 & 4.8753 & 4.6377 & 2.2327 & 3.7981 & 5.9333 \\
\hline \hline CC (HPF) & 0.9467 & 0.9957 & 0.9838 & 0.8800 & 0.9722 & 0.9777 \\
\hline
\end{tabular}

Table 2: Evaluation criteria (the correlation coefficients and ERGAS) for Alexandria data set 2 (Urban area) 